\title{
The mobilization, recruitment and contribution of bone marrow-derived endothelial progenitor cells to the tumor neovascularization occur at an early stage and throughout the entire process of hepatocellular carcinoma growth
}

\author{
HAITAO ZHU ${ }^{1,3^{*}}$, QIANWEN SHAO ${ }^{4 *}$, XITAI SUN ${ }^{1}$, ZHENGMING DENG $^{1}$, \\ XIANWEN YUAN $^{1}$, DECAI YU ${ }^{1}$, XIANG ZHOU ${ }^{2}$ and YITAO DING ${ }^{1}$

\begin{abstract}
${ }^{1}$ Institute of Hepatobiliary Surgery and Department of Hepatobiliary Surgery and ${ }^{2}$ Department of Pathology, The Affiliated DrumTower Hospital, School of Medicine, Nanjing University; ${ }^{3}$ Department of General Surgery, Institute of Oncology and The Affiliated Cancer Hospital of Nanjing Medical University, Jiangsu Cancer Hospital; ${ }^{4}$ Department of Oncology,

The First Affiliated Hospital, Nanjing Medical University, Nanjing, Jiangsu Province, P.R. China
\end{abstract}

Received February 17, 2012; Accepted May 11, 2012

DOI: $10.3892 /$ or.2012.1944

\begin{abstract}
Obvious neovascularization is a key feature of hepatocellular carcinoma (HCC) and the status of neovascularization in $\mathrm{HCC}$ is closely correlated with the tumor growth and patient prognosis. The actual effect of current antivascular treatment including embolization to $\mathrm{HCC}$ is not satisfactory. Compensatory angiogenesis is one of the primary causes responsible for failure of antiangiogenic therapy. Bone marrowderived endothelial progenitor cells (BM-EPCs) are considered as important building blocks for adult neovascularization. However, the role of mobilized BM-EPCs in HCC remains unknown. In this study, $\mathrm{GFP}^{+}-\mathrm{BM}$ orthotropic $\mathrm{HCC}$ mice were established to investigate whether BM-EPCs are involved in $\mathrm{HCC}$-induced neovascularization. We found that a large number of BM-EPCs were mobilized into the circulation with the development of $\mathrm{HCC}$, recruited into the $\mathrm{HCC}$ region and incorporated into the vascular endothelium directly by differentiation into vascular endothelial cells, including sinus, capillary vessels and great vessels. Dynamic observation revealed that the mobilization and the incorporation of BM-EPCs into different types of vessels were present in early phases and throughout the whole process of HCC growth. The proportion of BM-EPCs in vessels increased gradually, from 17 to $21 \%$ with tumor growth. Moreover, injected GFP ${ }^{+}$-EPCs also specifically homed to tumor
\end{abstract}

Correspondence to: Dr Yitao Ding, Institute of Hepatobiliary Surgery, The Affiliated DrumTower Hospital, Nanjing University, 210009 Nanjing, Jiangsu Province, P.R. China

E-mail: drdingyitao@yahoo.com.cn

*Contributed equally

Key words: hepatocellular neoplasms, neovascularization, endothelial progenitor cells tissue and incorporated into tumor vessels directly. In this initial study, we demonstrated that BM-EPCs play a prominent role in HCC neovascularization. Blockade of BM-EPC-mediated vasculogenesis may improve the efficacy of current anti-vascularization therapy for patients with HCC.

\section{Introduction}

Neovascularization is vital for progressive multiplication, metastasis and the recurrence of malignant tumors. Since Asahara et al isolated endothelial progenitor cells (EPCs) from human peripheral blood in 1997 (1), there is a growing body of evidence supporting the notion that adjacent activated endothelial cell sprouting is not a unique manner to form new blood vessels. Bone marrow derived-EPCs (BM-EPCs) have the capacity to stimulate the initiation and maintenance of angiogenic processes by integrating into developing vasculature under physiological and pathological conditions (2,3). EPCs resemble embryonic angioblasts which characteristically migrate, proliferate and differentiate into vascular endothelial cells (VECs) (4). In general, BM-EPCs can be identified as cells that simultaneously express cell surface markers CD133, CD34, and VEGFR2 $(5,6)$. Broadly, any bone marrow derived cells (BMDCs) that possess the potential of integrating into vessel walls by differentiating into VECs can be defined as endothelial progenitor cells/ endothelial precursor cells (EPCs) (7).

$\mathrm{HCC}$ is among the most vascularized tumors and the degree of vascularization correlates directly with prognosis in HCC (8). However, the actual effect of antivascular treatment including embolization to $\mathrm{HCC}$ has not reached the expectations. A thorny problem is the rapid development of new collateral circulation (9). There must be certain compensatory mechanisms that promote angiogenesis during therapy. With respect to cellular mechanisms, growing attention is being paid to the role of BM-EPCs in tumor angiogenesis (10-12). However, the degree of contribution by EPC to vasculature vary highly with tumor 
type in different studies, from substantial to zero, and the role of EPC, in particular, remains somewhat controversial $(13,14)$. Although increased circulating EPC levels have been reported in patients with HCC (15-17), up to now, there is no report providing direct evidence that BM-EPCs are involved in the neovascularization of $\mathrm{HCC}$, and its impact on angiogeneis in HCC remains uncertain.

Thus we established orthotropic HCC mice, observed the dynamic changes of circulating BM-EPCs, analyzed when the mobilization of BMCs starts and how long this process takes, examined whether BM-EPCs contributed to tumor blood vessels directly, and calculated the proportion of BM-EPCs in vessels. All of this information not only is extremely important in order to evaluate the impact of BM-EPCs on the neovascularization of HCC, but also may have important value to perfect the current anti-vascular strategy to patients with HCC.

\section{Materials and methods}

Isolation, culture, identification of $\mathrm{GFP}^{+} \mathrm{EPCs}$. GFP'EPCs were obtained according to the literature (18). Briefly, BM derived mononuclear cells from GFP transgenic C57BL/6 mice were collected through density gradient centrifugation and cultured in fibronectin coated dishes containing EGM-2 (Lonza, USA). After $48 \mathrm{~h}$, non-adherent cells were collected and cultured continually. All experiments were performed with second passage cells. The tube formation ability of BM-EPCs was observed within 24-h culture in matrigel (BD Biosciences, USA) (19). BM-EPC phenotypes were identified by flow cytometry (FCM) analysis. PE-conjugated anti-CD133, PECy7-conjugated anti-CD34, and PECy7-conjugated anti-VEGFR2 (eBioscience, USA) were applied. Appropriate fluorochrome-conjugated isotype was used as control. The $\mathrm{CD}_{133}{ }^{+} \mathrm{CD} 34^{+}$cells and $\mathrm{CD} 133^{+}{ }^{+}$EGFR2 ${ }^{+}$cells were classified as EPCs $(20,21)$. The animal research ethics committee of our hospital approved the protocol. All mice were sourced from the national genetically engineered mouse resources bank in China.

Orthotropic HCC model. In order to trace BM cells (BMCs), $\mathrm{GFP}^{+} \mathrm{BM}-\mathrm{C} 57 \mathrm{BL} / 6$ mice were established by transplanting whole BMCs of GFP-transgenic mice (22) (Fig. 1A). Four weeks later, FCM analysis was used to confirm full BM recovery. Orthotropic HCC mice were induced by an intrahepatic injection of $1 \sim 2 \times 10^{5} \mathrm{H} 22$ hepatoma cells (23) (gift from Dr Rutian Li, Institute of Oncology, Nanjing University, China). The control group received the same volume of PBS. To further confirm the role of EPCs on tumor angiogenesis, GFP EPCs $\left(1-2 \times 10^{6}\right)$ cultured in vitro were injected into orthotropic HCC nude mice via tail vein every $12 \mathrm{~h}$ for 3 days from day 7 after modeling (24). At day 14, mice liver, lung, kidney, pancreas, and stomach were examined to observe the distribution of injected $\mathrm{GFP}^{+} \mathrm{EPCs}$.

Flow cytometry. Peripheral blood $(300 \mu \mathrm{l})$ was obtained on days 3, 7, 14, and 21 after building orthotropic HCC mice. Mononuclear cells were separated and incubated for $30 \mathrm{~min}$ at $4^{\circ} \mathrm{C}$ using PE-conjugated anti-CD133, PE-conjugated antiCD34, and PE-conjugated anti-VEGFR2 (eBioscience, USA). Appropriate fluorochrome-conjugated isotypes were used as controls.
ELISA. Serum vascular endothelial growth factor (VEGF) and platelet-derived growth factor (PDGF) in HCC mice were tested using an ELISA kit (Abcam) following the collection of sera at the same time-points as stated above.

Immunofluorescence. By day 14 after establishing the GFP'BMorthotropic HCC model, $2 \mu \mathrm{m}$ frozen sections of mouse livers were cut and then incubated with primary antibodies overnight at $4^{\circ} \mathrm{C}$ : rat anti-mouse CD31 (1:800, eBioscience, USA). After washing with PBS, slices were incubated with secondary antibodies: Alexa Fluor 488-conjugated rabbit anti-rat IgG antibody (1:1000, Molecular Probes, USA). DAPI was used to dry the nucleus. To quantify the proportion of BM-EPCs in vessels, on days 7 and 21 after modeling, anti-CD31 immunofluorescence stained sections were analyzed by counting the number of $\mathrm{GFP}^{+}$ VECs and total VECs in 15 random high-power fields (21). The quantitative contribution of BM-EPCs to vessels was expressed as a percentage.

Immunohistochemistry. Sections of mouse livers $(2 \mu \mathrm{m})$ were cut as described above and then incubated with primary antibodies overnight at $4^{\circ} \mathrm{C}$ : rabbit anti-mouse ICAM1 (1:50, Protein Tech Group, USA), VCAM1 (1:200, Santa Cruz, USA), and VEGF (1:1000, Abcam). A secondary antibody was labeled using horseradish peroxidase. Positive reactions were visualized using diaminobenzidine solution followed by counterstaining with hematoxylin. Negative controls were obtained by substituting the primary antibodies with PBS.

Western blot analysis. Proteins $(100 \mu \mathrm{g})$ from each sample were subjected to $10 \%$ SDS-PAGE. Target protein levels were measured by immunoblotting with the corresponding antibodies: rabbit anti-mouse actin (1:800, Beyotime, China), rabbit anti-mouse ICAM1 (1:1000, Santa Cruz), rabbit anti-mouse VEGF (1:1000) and goat anti-mouse VCAM1 (1:1000, Abcam). They were then incubated for $30 \mathrm{~min}$ with secondary antibodies. Bands were visualized using enhanced chemiluminescence (Thermo, USA).

Real-time PCR. The levels of expression of BM-EPCs marker gene in tumor tissues (TF) and tumor-free tissues (TT) were determined using real-time PCR. RNA was extracted from the livers with TRIzol (Invitrogen, USA). The sequences of primers were synthesized by Takara (Takara, Japan): CD133 (5'-AAC GTG GTC CAG CCG AAT G-3', 5'-TCC CAG GAT GGC GCA GAT A-3'), CD34 (5'-ACC CAC CGA GCC ATA TGC TTA C-3', 5'-GAT ACC CTG GGC CAA CCT CA-3'), VEGFR2, (5' AGG GTG GTC CAG CCG AAT G-3', 5'TCC CAG GAT GGC GCA GAT A-3') and $\beta$-actin (5'-CAT CCG TAA AGA CCT CTA TGC CAA C-3', 5'-ATG GAG CCA CCG ATC CAC A-3'). PCR reactions were performed using a SYBR Premix Ex Taq Kit (Takara, Japan) and the Stratagene QPCR System (Bioscience, USA). Each sample was measured in duplicate. The relative levels of expression were determined by DDCt normalized to endogenous controls ( $\beta$-actin).

Statistical analysis. Numeric data were presented as mean \pm SD. t-tests and ANOVA were used to analyze the normal distribution data. All statistical analysis was performed using SPSS 15.0. Values of $\mathrm{P}<0.05$ were considered significant. 
A

harvest GFP BMCs

9Gy irradiated

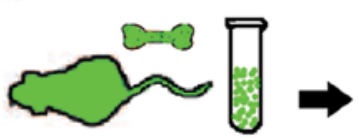

GFP transgenic mice

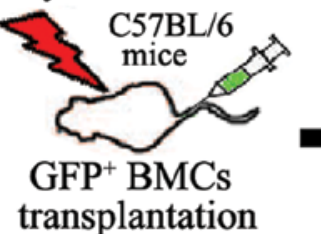

only BMCs

express GFP

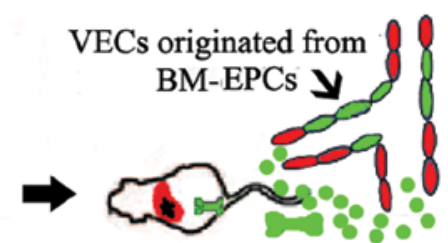

transplantation

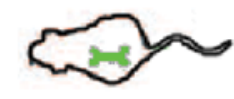

$\mathrm{BM}$ reconstitution

$\mathrm{GFP}^{+} \mathrm{BM}$ HCC mice
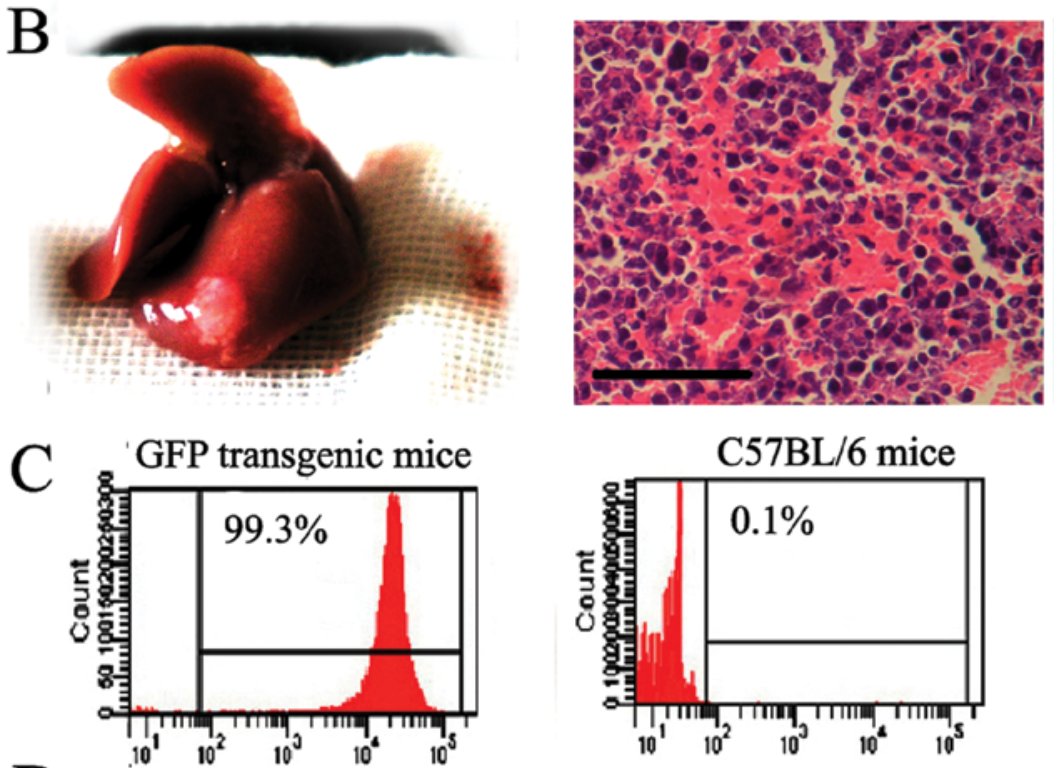

$D$

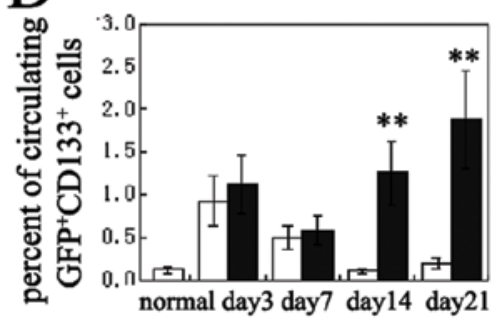

$\mathrm{E}$
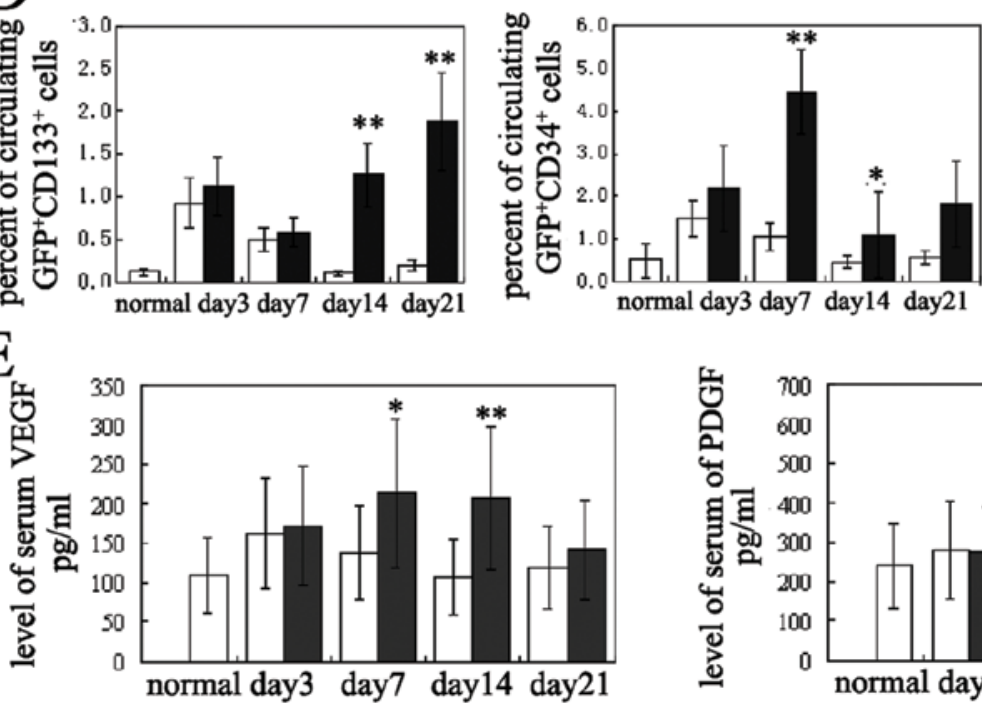

C57BL/6 mice
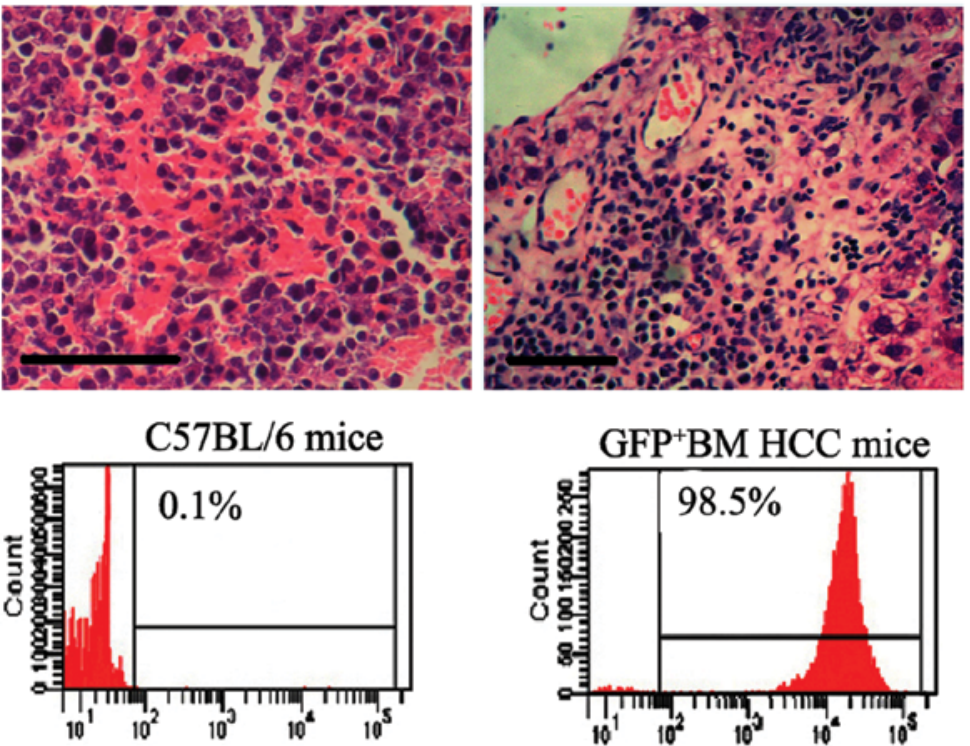

$\mathrm{GFP}^{+} \mathrm{BM} \mathrm{HCC}$ mice
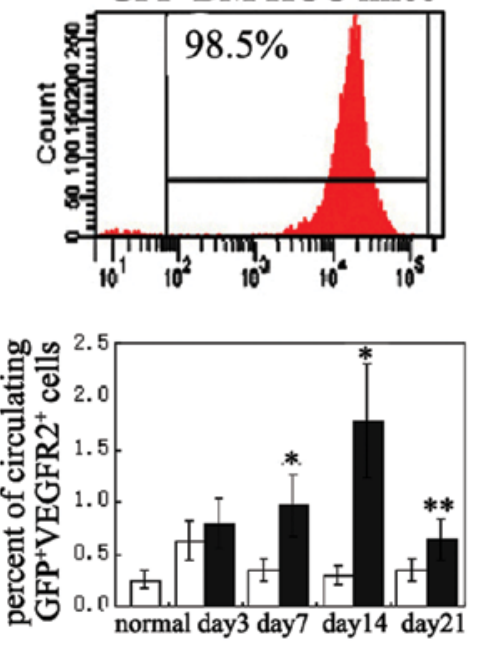

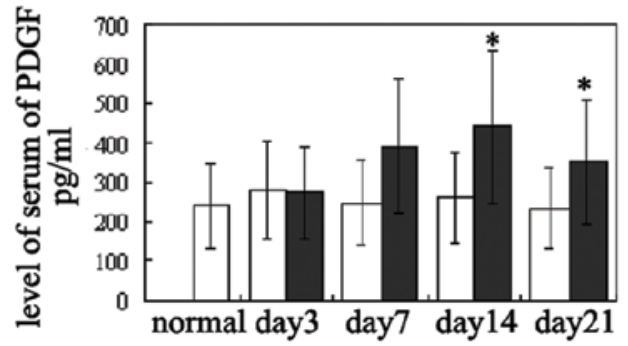

Figure 1. BM-EPCs were mobilzed into ciculation in orthotropic HCC mice. (A) A schematic and flow chart shows how GFP ${ }^{+}$-BM orthotropic HCC mice were established to assess the contribution of BM-EPCs to HCC induced neovascularization. (B) GFP+BM orthotopic HCC mice with classic pathological characteristics. 1, The gross change of liver in $\mathrm{GFP}^{+} \mathrm{BM}$-orthotopic $\mathrm{HCC}$ mice, 2 , Intratumor hemorrhage. 3, Intratumor neovasculariztion, bar, $10 \mu \mathrm{m}$ (paraffin, $\mathrm{H} \& \mathrm{E}, 2 \mu \mathrm{m}$ ). (C) FCM analysis of GFP positive rate of BMCs in GFP transgenic mice, normal mice, and GFP+BM HCC mice, respectively. (D and E) Dynamic change of circulating BM-EPCs and serum VEGF, PDGF in $\mathrm{HCC}$ mice vs sham-operation group. The number of circulating $\mathrm{GFP}^{+} \mathrm{CD} 133^{+}$, $\mathrm{GFP}^{+} \mathrm{CD}^{+} 4^{+}$, $\mathrm{GFP}^{+}$VEGFR2 ${ }^{+}$cells, and the levels of serum VEGF, PDGF in HCC group increased significantly compared with those in sham-operation group. ${ }^{*} \mathrm{P}<0.05$; ${ }^{* *} \mathrm{P}<0.01$; normal, normal mice; $\mathrm{n}=8$. $\mathbf{\mathrm { a }}, \mathrm{HCC}$ mice group; $\square$, sham-operation group.

\section{Results}

The success of establishing the GFP-labeled BM-orthotropic HCC model. The pathological examinations demonstrated that $\mathrm{GFP}^{+} \mathrm{BM}$-orthotropic HCC mice retained classic clinicopathological characteristics (Fig. 1B). The high $\mathrm{GFP}^{+}$rate of circulating nucleated cells in the HCC model (>95\%) (Fig. 1C) suggested that nearly all BMCs express GFP stably, guaranteeing success in distinguishing BM-EPCs derived VECs from pre-existed VECs (Fig. 1A).

BM-EPCs were mobilized into circulation. In general BM-EPCs are believed to originate from hematopoietic stem cells and express stem cell antigens CD133, CD34, and VEGFR2 $(5,6)$. 

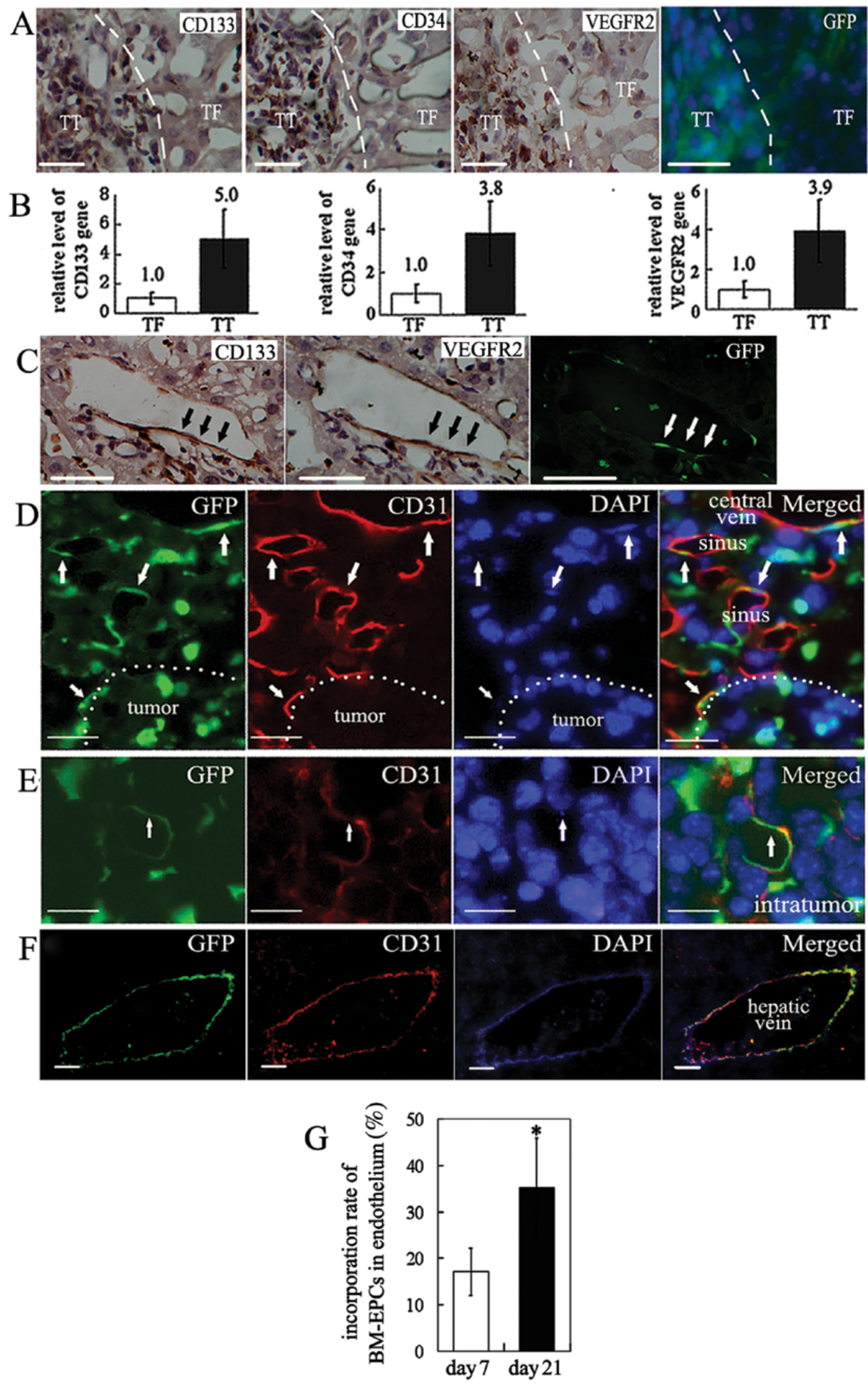

Figure 2. BM-EPCs were recruited and contribute to neovascularization in HCC. (A) Distribution of EPC marker phenotype CD133, CD34 and VEGFR2 antigens in TT and TF in three of four consecutive sections of HCC. Dotted lines represent the clusters of positively stained cells in immunohistochemistry sections. In serial sections, the distribution of BMCs expressing GFP and the expression of EPC marker antigen are consistent. The BMCs labeled by GFP appear green with the nuclei stained blue by DAPI. Positive staining appears brown (bar, $20 \mu \mathrm{m}$ ). (B) Relative level of CD133, CD34 and VEGFR2 gene in TT and TF ( $\mathrm{n}=7$ ). (C) $\mathrm{CD}_{133^{+}}$, VEGFR2 ${ }^{+}$cells incorporated into vascular endothelium of HCC liver. Arrows indicate CD133 $3^{+}$VEGFR2 $2^{+}$cells (bar, $15 \mu \mathrm{m}$ ). (D) BM-EPCs infiltration to peritumoral hepatic sinus and central veins (bar, $10 \mu \mathrm{m}$ ). (E) BM-EPCs infiltration to intratumoral vessels (bar, $5 \mu \mathrm{m}$ ). (F) BM-EPCs infiltration to hepatic veins (bar, $40 \mu \mathrm{m}$ ). Mature ECs expressing marker CD31 are stained red. BMCs tagged by GFP are stained green. Nuclei stained with DAPI appear blue. Arrows indicate CD31 ${ }^{+} \mathrm{GFP}^{+} \mathrm{VECs}$. (G) The incorporation rate of EPCs in $\mathrm{HCC}$ blood vessels at day 21 versus that at day $7 .{ }^{*} \mathrm{P}<0.01, \mathrm{n}=15$. 
While CD133, CD34 are expressed also in certain tumor cells, such as hepatic cancer stem cells $(25,26)$. Thus, to a certain degree, the number of circulating $\mathrm{GFP}^{+} \mathrm{CD} 133^{+}, \mathrm{GFP}^{+} \mathrm{CD} 34^{+}$ and $\mathrm{GFP}^{+} \mathrm{VEGFR} 2^{+}$cells can reflect the mobilized degree of BM-EPCs. FCM analysis showed that by day 7 after modeling, the mean percentages of circulating $\mathrm{GFP}^{+} \mathrm{CD} 34^{+}$ and $\mathrm{GFP}^{+} \mathrm{VEGFR} 2^{+}$cells in HCC mice were $0.97 \pm 0.29$ and $4.46 \pm 3.47 \%$, respectively, which were much higher than those in control mice $(0.35 \pm 0.14$ and $1.04 \pm 1.32 \%)$. By day 14 , the mean percentages of circulating $\mathrm{GFP}^{+} \mathrm{CD}_{133^{+}}, \mathrm{GFP}^{+} \mathrm{CD} 34^{+}$and $\mathrm{GFP}^{+}$VEGFR $2^{+}$cells in HCC mice were $1.26 \pm 0.70,1.77 \pm 1.35$ and $1.08 \pm 0.59 \%$, respectively, which increased dramatically compared with those in control mice $(0.12 \pm 0.15,0.31 \pm 0.24$, and $0.46 \pm 0.34 \%$ ). By day 21 , mean percentages of circulating $\mathrm{GFP}^{+} \mathrm{CD} 133^{+}, \mathrm{GFP}^{+} \mathrm{VEGFR} 2^{+}$cells $(1.88 \pm 0.98$ and $1.8 \pm 0.88 \%)$ were also elevated relative to control mice $(0.20 \pm 0.23$ and $0.55 \pm 0.19 \%$ ) (Fig. 1D). The ELISA results indicated that compared with a transient increase in the control, serum VEGF and PDGF maintained at a higher level were obviously induced by HCC (Fig. 1E).

$B M-E P C s$ were recruited and incorporated into vascular endothelium. The distribution of BM-EPCs was assessed based on the distribution of the specific surface antigens (CD133, CD34, and VEGFR2), combined with GFP (the tracer of BMCs) in the serial sections. Consecutive immunohistochemistry data showed that the BM-EPCs surface markers CD133, CD34, VEGFR2, and GFP antigens were mostly concentrated on TT compared to TF (Fig. 2A). Quantity PCR showed the levels of CD133, CD34 and VEGFR2 genes in TT were also much higher than that in TF (Fig. 2B). Based on fluorescence microscopy, these positive cells were from BM as evidenced by GFP in serial sections. At high magnifications, we found that some VECs co-expressed CD133, VEGFR2 and GFP, which is direct evidence that BM-EPCs melted into vascular endothelium by differentiating into VECs in situ (Fig. 2C).

$B M-E P C s$ contribute to the formation of different type vessels in $H C C$. It is very difficult to quantitatively analyze the incorporation rate of classic endothelial progenitor cells into the endothelium because the stem cells would be undetectable after the loss of their surface markers during differentiation. Based on the broad sensing of BM-EPCs $(7,21)$, the GFP combined with the EC-specific antigen CD31 (18) makes it easy to identify whether the BM-EPCs incorporate into the vascular endothelium in the $\mathrm{GFP}^{+} \mathrm{BM}$ HCC mice (Fig. 1A). In this study, we found that the $\mathrm{CD} 1^{+} \mathrm{GFP}^{+}$cells existed not only in the peripheral vessels but also in the intratumoral vessels and in vessel walls of different sizes, such as sinusoids, central veins and hepatic veins (Fig. 2D-F). These $\mathrm{CD} 31^{+} \mathrm{GFP}^{+}$cells offer direct evidence that the BM-EPCs contribute to the $\mathrm{HCC}$-induced neovascularization. Additionally, more BM-EPCs incorporated into the vascular endothelium in the HCC tissue during tumor progression, as observed by the higher rate of incorporation of BM-EPCs in all VECs, at day 21 $(35.3 \pm 21.2 \%)$ than at day 7 (17.1 $\pm 8.9 \%)$ (Fig. 2G). This indicated that BM-EPCs are needed and play an important role in HCC angiogenesis.

Injected $\mathrm{GFP}^{+} \mathrm{EPCs}$ specifically home to tumor and incorporate into blood vessels. In culture, GFP EPCs gradually attached onto fibronectin-coated plates, and adopted the shape of cobblestones, a characteristic morphology of EPCs. Two weeks later, these cells proliferated rapidly and could form tube-like structures on matrigel, a functional feature of EPCs (Fig. 3B). FCM analysis revealed $85 \%$ of cultured cells were CD133 ${ }^{+}$VEGFR2 ${ }^{+}$ cells or $\mathrm{CD} 133^{+} \mathrm{CD} 34^{+}$cells (Fig. $3 \mathrm{C}$ ), which indicated success in obtaining GFP'EPCs. At day 7 after $\mathrm{GFP}^{+} \mathrm{EPC}$ injection, GFP positive cells were found scarcely distributed in the organs examined, lungs, kidneys, pancreas, stomach, except for within the liver tumor mass, where injected $\mathrm{GFP}^{+} \mathrm{EPCs}$ were highly concentrated (Fig. 3D). At high magnification, the $\mathrm{GFP}^{+} \mathrm{CD} 31^{+}$ cells were found in tumor vascular endothelium, which is direct, intuitive evidence that BM-EPCs contributed to the formation of new tumor vessels (Fig. 3E and F).

Up-regulated expression of ICAM1, VCAM1, and VEGF in HCC tissue. Immunohistochemistry and western blot results showed that the expression of ICAM1, VCAM1, and VEGF in TT was much higher than it was in TF (Fig. 4).

\section{Discussion}

Neovascularization is a crucial factor for $\mathrm{HCC}$ to grow and metastasize. The role of BM-EPCs on tumor angiogenesis is still debated $(13,14)$. Even though the mobilization of BM-EPCs has been demonstrated in HCC $(15,16)$, the precise role of BM-EPCs in HCC angiogenesis is not well understood. Exploring the role of BM-EPCs on HCC neovascularization, on the one hand, is to indentify whether BM-EPCs contribute to the HCC-induced neovascularization in theory, on the other hand may have implications for renovation of current therapeutic strategy to patients with HCC in practice.

Previous clinical study showed that BM-EPCs mainly concentrated on adjacent non-malignant liver tissue rather than HCC tissue (17). Though the exact distribution of mobilized BM-EPCs in target organ is still in dispute $(27,28)$, we have to accept the notion that recruitment into target tissues is a prerequisite for the effectiveness of BM-EPCs. Moreover, according to the theory that further tumor growth is accompanied by the formation of tumor vessels, HCC tissue should contain more BM-EPCs, in accordance with HCC neovasculature development. The image data from serial sections substantiate our hypothesis. The marker antigens CD133, CD34, VEGFR2, and GFP that are the hallmark of BM-EPCs, were mainly concentrated in HCC tissues (Fig. 2A), as measured by quantitative PCR (Fig. 2B). Moreover, also injected BM-EPCs homed to TT with high specificity (Fig. 3D). To understand the homing mechanism, the expression of cell adhesion molecules ICAM1, VCM1 and VEGF in TT and TF was examined. We found increased serum VEGF, PDGF (Fig. 1E) and that VEGF, ICAM1 and VCAM1 were predominately expressed in TT (Fig. 4). ICAM-1 is considered the main cellular adhesive molecule and VEGF is the most essential mobilization factor $(29,30)$. We reasonably inferred that with HCC proliferation, the BM-EPCs were mobilized into circulation by $\mathrm{BM}$ mobilization factors released from HCC and subsequently entered into liver via the blood stream. The cells finally were arrested on HCC tissues specifically due to the induction of a high local content of adhesive molecules. The main reasons for the conflicting conclusions may stem from the following: a) HCC mice lack a background 


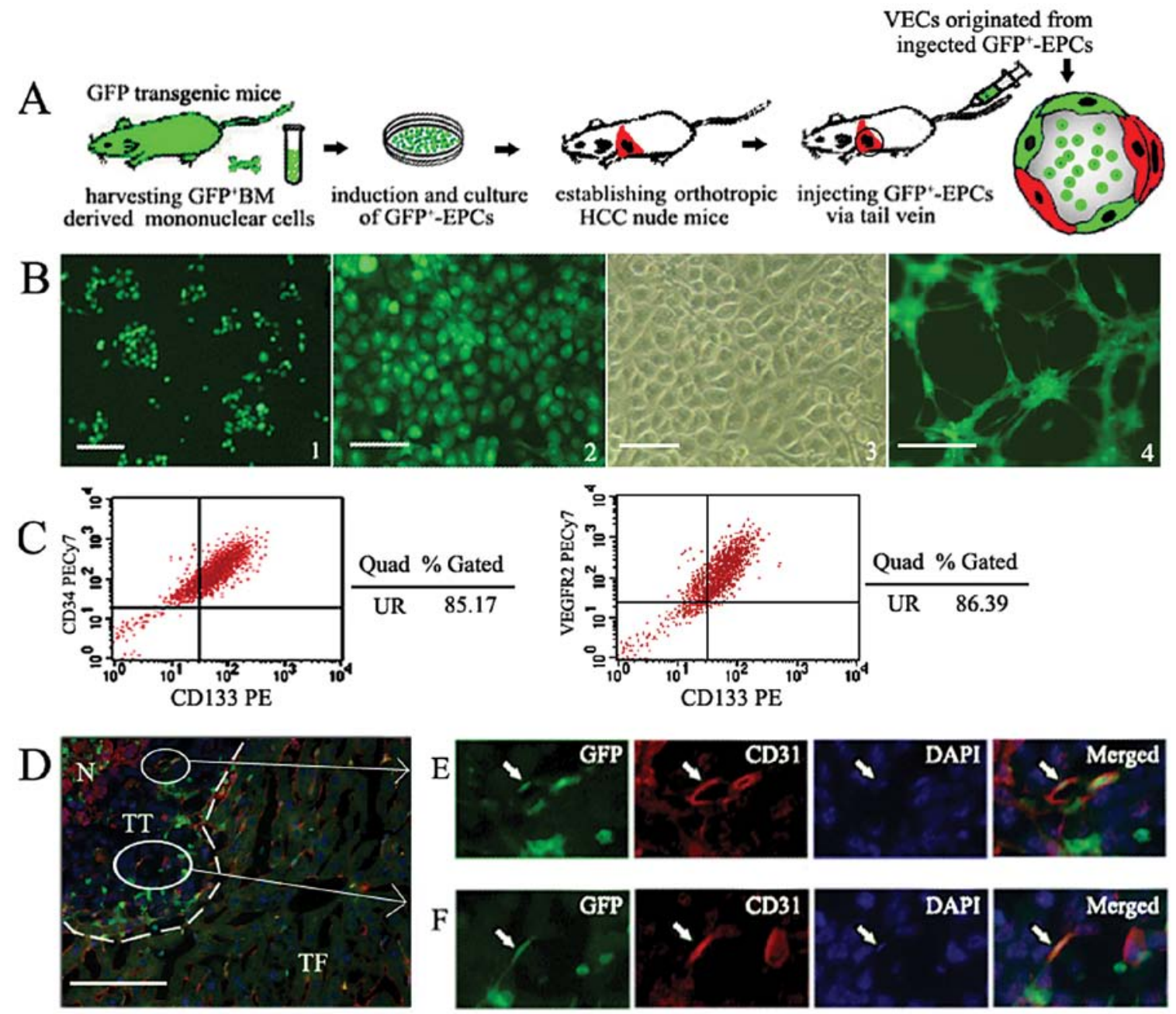

Figure 3. Injected GFP BM-EPCs specifically home to tumor and incorporate into tumor vessels. (A) A schematic and flow chart shows BM-EPCs cultured in vitro were injected into orthotropic HCC nude mice in order to further confirm that BM-EPCs were specifically recruited and incorporate into endothelium of tumor vessels. (B) Isolation, culture and identification of GFP'EPCs. 1, GFP'-mononuclear cells obtained form GFP transgenic mice cultured in the first week. 2, The photograph of second passage GFP+EPCs cultured in EGM-2, observed under fluorescent inverse microscopy. 3, A representative phase-contrast photograph of second passage GFP'EPC, identified as a well-circumscribed monolayer of cobblestone-appearing cells. 4, GFP+EPCs form capillary-like structures within 24-h culture in matrigel (bar, $200 \mu \mathrm{m}$ ). (C) Flow cytometric analysis of GFP'EPCs cultured in vitro. (D) Injected GFP'EPCs were recruited into HCC tissue. Dotted lines show the tumor position. $\mathrm{N}$, tumor necrosis area. (E and F) The higher magnification of the area denoted by a rectangle in (D). At high magnification, the GFP $\mathrm{CD}^{+} 1^{+}$ cells were found in vascular endothelium in HCC tissue. Mature VECs expressing marker CD31 are stained red. BMCs tagged by GFP are stained green. Nuclei stained with DAPI appear blue. Arrows indicate VECs co-expressing CD31 and GFP (frozen section, $2 \mu \mathrm{m}$, bar, $25 \mu \mathrm{m}$ ).

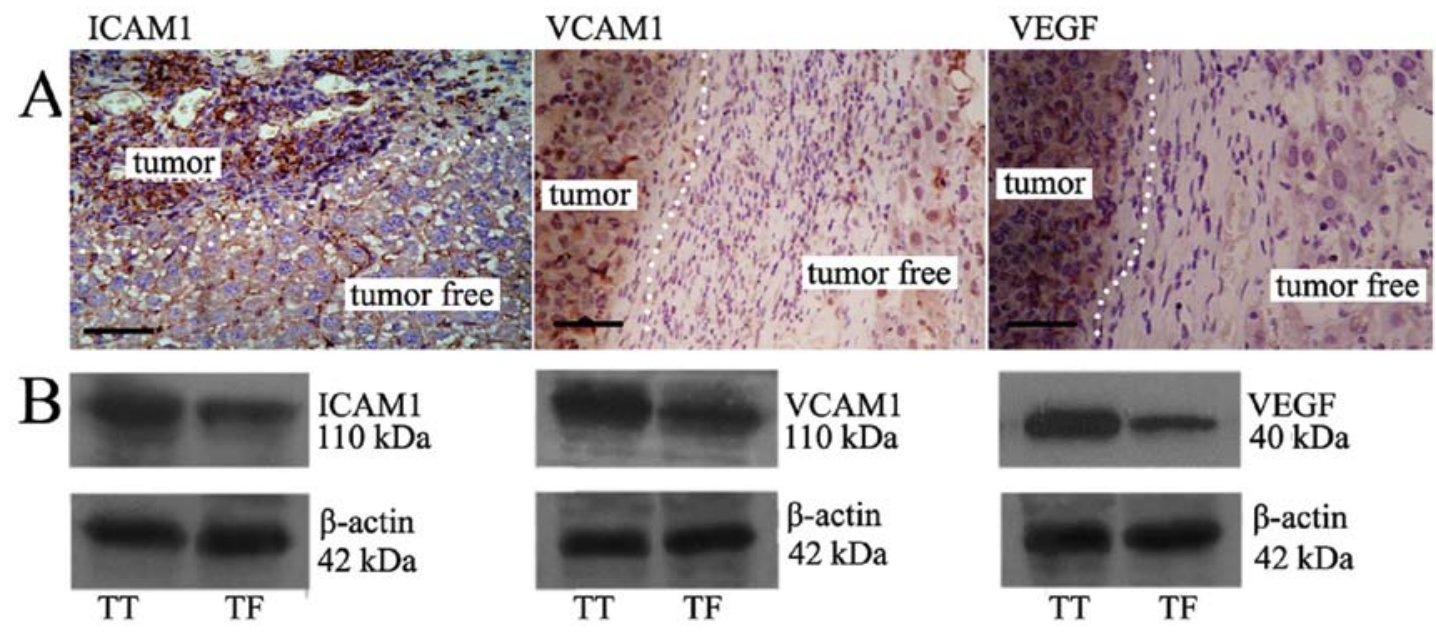

Figure 4. The expression of ICAM1, VCAM1, VEGF in TT vs TF. (A) Immunohistochemistry shows the expression of ICAM1, VCAM1, VEGF in TT was much more than that in TF. Dotted lines show the tumor position. Brown particles, positive products of ICAM1, VCAM1, VEGF protein, respectively. Bar, $20 \mu \mathrm{m}$ (paraffin, $2 \mu \mathrm{m}$ ). (B) Western blotting demonstrated that expression of ICAM1, VCAM1, VEGF in TT was higher than that in TF. The upper bands are the protein, respectively; the lower are the internal standard. 
of chronic hepatitis and/or cirrhosis-precancerous diseases with angiogenesis $(31,32), \mathrm{b}) \mathrm{HCC}$ in the present study is as a result of implanted tumors rather than from spontaneous tumors, c) rapid tumor proliferation results in augmented blood supply within a short time or d) different types or stages of cancer may have led to the different conclusions. Despite these speculations, our results may reflect to some degree the natural link between HCC growth and BM-EPCs. Two effects of recruited BM-EPCs on neovascularization were found. First, BM-EPCs cells were incorporated into vascular endothelium directly, and second, proangiogenic factors were secreted $(33,34)$.

The current debate as to when during progressive tumor growth BM-EPCs are mobilized and recruited into the tumor continues. Some believed that the vasculogenesis of BM-EPCs is a late event (35), whereas others have taken the view that BM-EPCs were incorporated for a brief period during early phases ( $<2$ weeks) of tumor growth. The longerterm tumor vascular endothelium was derived from nearby host vessels (36). However, in our study, the contribution of BM-EPCs to tumor vessels was detectable by day 7 , by which point the proportion of BM-EPCs in all VECs had already reached $17 \%$. Thus, the recruitment of mobilized BM-EPCs to HCC tissues must arise before this time-point. Obviously, the mobilization of BM-EPCs began much earlier. Moreover, the proportion of BM-EPCs in vessels gradually increased to $35 \%$ by day 21 along with tumor growth (Fig. $2 \mathrm{G}$ ). We believe that the contribution of BM-EPCs to neovascularization is not the sole feature of advanced tumors but rather an integral part of tumor development that becomes activated during the 'angiogenic switch' and is required for early, premalignant lesions to progress to frank tumors. In fact, the premalignant changes of HCC such as cirrhosis or hepatitis virus infection have tangible angiogenesis $(31,32)$. After tumor formation, neovascularization becomes the outstanding characteristic of HCC and determines tumor progression and patient prognosis.

Although Asahara et al (2) demonstrated the presence of circulating BM-EPCs, the role of BM-EPCs in neovasculariztion has remained controversial. Some even demonstrated that EPCs were not required for tumor growth at all $(14,37)$. While in HCC, BM-EPCs were specifically recruited into TT and truly incorporated into peritumoral and intratumoral vessels, involving different types of vasculatures such as sinus, central veins, microvasculature, and large vessels (Fig. 2D-F). Moreover, the BM-EPCs injected via vein were also specifically rested on TT and contributed to the formation of tumor new vessels (Fig. 3D). The BM-EPCs may have also maintained HCC neovascularization, because their proportion in vessels increased gradually along tumor growth (Fig. 2G). In HCC mice, we found that BM-EPCs mainly integrated into the network of pre-existing vessels rather than forming an entirely new vascular endothelium. Increased vascular caliber was usually detectable earlier than the morphological signs of tumors (38). We infer that there might exist two patterns of how BM-EPCs contribute to tumor neovascularization: one is dilatation in diameter and the other is an extension in length. In large vessels, mobilized BM-EPCs supplied endothelial cells in order to meet the demand to enlarge the inner diameter to increase blood flow. In microvasculature, BM-EPCs can make capillaries longer and wider. Obviously, with the deposition of matrices, contribution of pericytes (39), and dilatation in diameter, these capillaries would soon become new, larger vessels that carry more blood to support tumor rapid proliferation.

The dependence of tumor growth on angiogenesis has been generally acknowledged, however, the actual effect of antivascular treatment including embolization to patients with HCC remains limited. A thorny problem is the rapid development of new collateral circulation (9). It has been reported that treated tumors dramatically increase in serum VEGF $(40,41)$ and circulating BM-EPCs $(42,43)$. Obviously, the further aggravation of tumor metabolic acidosis, hypoxia, and necrosis that result from ischemia would in turn lead to more soluble factors being released into blood. Subsequently, more BM-EPCs would be mobilized. Therefore, even though the tumor vasculature could be damaged, BM-EPCs provide an alternative source of VECs that contribute to formation of new vessels in order to compensate for this blood supply loss. BM-EPCs seem to play an important part in the compensatory cellular and molecular mechanisms that inhibit the efficiency of present treatment strategies. Thus, we should seriously consider inhibiting the mobilization and recruitment of BM-EPCs as a therapeutic target in HCC treatment.

In concusion, the presented data indicated that BM-EPCs were recruited specifically and contributed to neovascularization in HCC. This process began at an early stage, and may continue throughout the whole process of HCC growth. Moreover, the injected BM-EPCs homed to tumors with significantly higher specificity and also contribute to the formation of tumor vessels. These findings suggest that BM-EPCs are needed and play an important role in angiogenesis in HCC. Thus BM-EPCs might serve as biomarkers for predicting the progression or recurrence of HCC, and blocking the BM-EPCs mediated angiogenesis may help improve the efficacy of current therapies for HCC patients.

\section{Acknowledgements}

We thank Fan Xiangshan, Chen Jun, Xie Lili for excellent technical assistance and Cui Fengming for help in carrying out the animal experiments. This study was supported by National Natural Science Fund (30972904) and 135 Key Clinical Center of Institutes of Health in Jiangsu Province (SK200215).

\section{References}

1. Asahara T, Murohara T, Sullivan A, et al: Isolation of putative progenitor endothelial cells for angiogenesis. Science 275: 964-967, 1997.

2. Asahara T, Masuda H, Takahashi T, et al: Bone marrow origin of endothelial progenitor cells responsible for postnatal vasculogenesis in physiological and pathological neovascularization. Circ Res 85: 221-218, 1999.

3. Mund JA and Case J: The role of circulating endothelial progenitor cells in tumor angiogenesis. Curr Stem Cell Res Ther 6: 115-121, 2011.

4. George AL, Bangalore-Prakash P, Rajoria S, et al: Endothelial progenitor cell biology in disease and tissue regeneration. $\mathrm{J}$ Hematol Oncol 24: 24, 2011.

5. Yoder MC: Defining human endothelial progenitor cells. J Thromb Haemost 7: 49-52, 2009.

6. Doyle B, Metharom P and Caplice NM: Endothelial progenitor cells. Endothelium 13: 403-410, 2006.

7. Chao $\mathrm{H}$ and Hirschi KK: Hemato-vascular origins of endothelial progenitor cells? Microvasc Res 79: 169-173, 2010.

8. Zhu AX, Duda DG, Sahani DV, et al: HCC and angiogenesis: possible targets and future directions. Nat Rev Clin Oncol 8: 292-301, 2011. 
9. Kim HC, Chung JW, Lee W, et al: Recognizing extrahepatic collateral vessels that supply hepatocellular carcinoma to avoid complications of transcatheter arterial chemoembolization. Radiographics 25: S25-S39, 2005.

10. Lyden D, Hattori K, Dias S, et al: Impaired recruitment of bonemarrow-derived endothelial and hematopoietic precursor cells blocks tumor angiogenesis and growth. Nat Med 7: 1194-1201, 2005.

11. Mellick AS, Plummer PN, Nolan DJ, et al: Using the transcription factor inhibitor of DNA binding 1 to selectively target endothelial progenitor cells offers novel strategies to inhibit tumor angiogenesis and growth. Cancer Res 70: 7273-7282, 2010.

12. Rigolin GM, Maffei R, Rizzotto L, et al: Circulating endothelia cells in patients with chronic lymphocytic leukemia: clinicalprognostic and biologic significance. Cancer 116: 1926-1937, 2010.

13. Hagensen MK, Raarup MK, Mortensen MB, et al: Circulating endothelial progenitor cells do not contribute to regeneration of endothelium after murine arterial injury. Cardiovasc Res 93: 223-231, 2012.

14. Wickersheim A, Kerber M, de Miguel LS, et al: Endothelial progenitor cells do not contribute to tumor endothelium in primary and metastatic tumors. Int J Cancer 125: 1771-1777, 2009.

15. Sieghart W, Fellner S, Reiberger T, et al: Differential role of circulating endothelial progenitor cells in cirrhotic patients with or without hepatocellular carcinoma. Dig Liver Dis 41: 902-906, 2009.

16. Ho JW, Pang RW, Lau C, et al: Significance of circulating endothelial progenitor cells in hepatocellular carcinoma. Hepatology 44: 836-843, 2006.

17. Yu D, Sun X, Qiu Y, et al: Identification and clinical significance of mobilized endothelial progenitor cells in tumor vasculogenesis of hepatocellular carcinoma. Clin Cancer Res 13: 3814-3824, 2007.

18. Sangidorj O, Yang SH, Jang HR, et al: Bone marrow-derived endothelial progenitor cells confer renal protection in a murine chronic renal failure model. Am J Physiol Renal Physiol 299: F325-F335, 2010.

19. Hu J, Dong A, Fernandez-Ruiz V, et al: Blockade of Wnt signaling inhibits angiogenesis and tumor growth in hepatocellular carcinoma. Cancer Res 69: 6951-6959, 2009.

20. Peichev M, NaiyerAJ, Pereira D, et al: Expression of VEGFR-2 and $\mathrm{AC} 133$ by circulating human $\mathrm{CD} 34^{+}$cells identifies a population of functional endothelial precursors. Blood 95: 952-958, 2000.

21. Massa M, Rosti V, Ramajoli I, et al: Circulating CD34+, CD133+, and vascular endothelial growth factor receptor 2-positive endothelial progenitor cells in myelofibrosis with myeloid metaplasia. J Clin Oncol 23: 5688-5695, 2005.

22. Suriano R, Chaudhuri D, Johnson RS, et al: 17Beta-estradiol mobilizes bone marrow-derived endothelial progenitor cells to tumors. Cancer Res 68: 6038-6042, 2008.

23. Schmitz V, Tirado-Ledo L, Tiemann K, et al: Establishment of an orthotopic tumor model for hepatocellular carcinoma and noninvasive in vivo tumour imaging by high resolution ultrasound in mice. J Hepatol 40: 787-791, 2004.

24. Ahn JB, Rha SY, Shin SJ, et al: Circulating endothelial progenitor cells (EPC) for tumor vasculogenesis in gastric cancer patients. Cancer Lett 288: 124-132, 2010

25. Ding W, Mouzaki M, You H, et al: CD133+ liver cancer stem cells from methionine adenosyl transferase 1A-deficient mice demonstrate resistance to transforming growth factor (TGF)-beta-induced apoptosis. Hepatology 49: 1277-1286, 2009.
26. Zhu Z, Hao X, Yan M, et al: Cancer stem/progenitor cells are highly enriched in $\mathrm{CD} 133^{+} \mathrm{CD} 44^{+}$population in hepatocellular carcinoma. Int J Cancer 126: 2067-2078, 2010.

27. Arbab AS, Pandit SD, Anderson SA, et al: Magnetic resonance imaging and confocal microscopy studies of magnetically labeled endothelial progenitor cells trafficking to sites of tumor angiogenesis. Stem Cells 24: 671-687, 2006.

28. Shirakawa K, Furuhata S, Watanabe I, et al: Induction of vasculogenesis in breast cancer models. Br J Cancer 87: 1454-1461, 2002.

29. Croll SD, Ransohoff RM, Cai N, et al: VEGF-mediated inflammation precedes angiogenesis in adult brain. Exp Neurol 187: 388-402, 2004

30. Gehling, U.M, Ergun S, Schumacher U, et al: In vitro differentiation of endothelial cells from AC133-positive progenitor cells. Blood 95: 3106-3112, 2000

31. Messerini L, Novelli L and Comin CE: Microvessel density and clinicopathological characteristics in hepatitis $\mathrm{C}$ virus and hepatitis B virus related hepatocellular carcinoma. J Clin Pathol 57: 867-871, 2004.

32. Pinzani M and Vizzutti F: Fibrosis and cirrhosis reversibility: clinical features and implications. Clin Liver Dis 12: 901-913, 2008.

33. Moon MH, Kim SY, Kim YJ, et al: Human adipose tissue-derived mesenchymal stem cells improve postnatal neovascularization in a mouse model of hindlimb ischemia. Cell Physiol Biochem 17: 279-290, 2006

34. Thomas J. O'Neill IV, Brian R, et al: Mobilization of bone marrowderived cells enhances the angiogenic response to hypoxia without transdifferentiation into endothelial cells. Circ Res 97: 1027-1035, 2005.

35. Hämmerling GJ and Ganss R: Vascular integration of endothelial progenitors during multistep tumor progression. Cell Cycle 5: 509-511, 2006.

36. Nolan D J, Ciarrocchi A, Mellick AS, et al: Bone marrow-derived endothelial progenitor cells are a major determinant of nascent tumor neovascularization. Genes Dev 12: 1546-1558, 2007.

37. He Y, Rajantie I, Ilmonen M, et al: Preexisting lymphatic endothelium but not endothelial progenitor cells are essential for tumor lymphangiogenesis and lymphatic metastasis. Cancer Res 64: 3737-3740, 2004.

38. Ryschich E, Schmidt J, Hammerling GJ, et al: Transformation of the microvascular system during multistage tumorigenesis. Int $J$ Cancer 97: 719-725, 2002.

39. Dar A, Domev H, Ben-Yosef O, et al: Multipotent vasculogenic pericytes from human pluripotent stem cells promote recovery of murine ischemic limb. Circulation 125: 87-99, 2012.

40. Poon RT, Lau C, Yu WC, et al: High serum levels of vascular endothelial growth factor predict poor response to transarterial chemoembolization in hepatocellular carcinoma: a prospective study. Oncol Rep 11: 1077-1084, 2004.

41. Wang B, Xu H, Gao ZQ, et al: Increased expression of vascular endothelial growth factor in hepatocellular carcinoma after transcatheter arterial chemoembolization. Acta Radiol 49: 523-529, 2008

42. Shaked Y, Henke E, Roodhart JM, et al: Rapid chemotherapyinduced acute endothelial progenitor cell mobilization: implications for antiangiogenic drugs as chemosensitizing agents. Cancer Cell 14: 263-273, 2008

43. Pircher A, Kähler CM, Skvortsov S, et al: Increased numbers of endothelial progenitor cells in peripheral blood and tumor specimens in non-small cell lung cancer: a methodological challenge and an ongoing debate on the clinical relevance. Oncol Rep 19: 345-352, 2008. 